\title{
Trypanosoma cruzi: Acute and long-term infection in the vertebrate host can modify the response to benznidazole
}

\author{
Sérgio Caldas ${ }^{a}$, Fabiane M. Santos ${ }^{a}$, Marta de Lana ${ }^{\text {a,b }}$, Lívia F. Diniz ${ }^{\mathrm{c}}$, \\ George L.L. Machado-Coelho ${ }^{\mathrm{a}, \mathrm{d}}$, Vanja M. Veloso ${ }^{\mathrm{a}, \mathrm{c}}$, Maria T. Bahia ${ }^{\mathrm{a}, \mathrm{c}, *}$ \\ a Núcleo de Pesquisas em Ciências Biológicas, Universidade Federal de Ouro Preto, Minas Gerais, Brazil

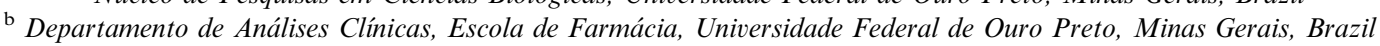 \\ ${ }^{\mathrm{c}}$ Departamento de Ciências Biológicas, Instituto de Ciências Exatas e Biológicas, Universidade Federal de Ouro Preto, Minas Gerais, Brazil \\ d Departamento de Farmácia, Escola de Farmácia, Universidade Federal de Ouro Preto, Minas Gerais, Brazil
}

Received 20 March 2007; received in revised form 27 June 2007; accepted 29 August 2007

Available online 7 September 2007

\begin{abstract}
We analyzed the influence of Trypanosoma cruzi maintenance in different hosts (dog and mouse) on its susceptibility to benznidazole treatment. Five T. cruzi stocks were isolated from dogs inoculated with Be-62 or Be-78 strain (both sensitive to benznidazole) $2-10$ years ago, and the benznidazole sensitivity was then determined using the mouse as experimental model. The different $T$. cruzi stocks obtained from long-term infected dogs showed 50-90\% drug resistance right after isolation. However, maintenance of these $T$. cruzi stocks in mice, by successive blood passages ( 2.5 years), led to either a decrease or stability of the drug resistance pattern and an increase in parasite virulence. We also demonstrated the effectiveness of the induction of parasitemia reactivation by cyclophosphamide immunosuppression in the evaluation of the response to the specific drug treatment.
\end{abstract}

(C) 2007 Elsevier Inc. All rights reserved.

Index Descriptors and Abbreviations: Trypanosoma cruzi; Chagas disease; Drug resistance; Chemotherapy; Cure control; Bz, benznidazole; Nf, nifurtimox

\section{Introduction}

The use of DNA probes targeted to T. cruzi kinetoplast DNA extracted from mummified Andean humans demonstrated that Chagas disease was highly prevalent in Southern Andean Coastal Area 9000 years ago (Aufderheide et al., 2004). Currently, 16-18 million people in Mexico, Central American and Latin American countries are infected with $T$. cruzi, and 40 million remain at risk of acquiring the disease (WHO, 2002).

Specific chemotherapy for Chagas disease was developed empirically about three decades ago but still remains very unsatisfactory. Currently available drugs are: a nitro-

\footnotetext{
* Corresponding author. Address: Departamento de Ciências Biológicas, Instituto de Ciências Exatas e Biológicas, Universidade Federal de Ouro Preto, Minas Gerais, Brazil. Fax: +55 3135591680.

E-mail address: mtbahia@nupeb.ufop.br (M.T. Bahia).
}

imidazole compound, benznidazole (Bz) (Rochagan, Radanil, Roche) and a nitrofuran compound, nifurtimox (Nf) (Lampit, Bayer, recently discontinued). According to clinical trials, the earlier the diagnosis and specific treatment the higher the chances of parasitological cure (Guedes et al., 2006). Differences in drug susceptibility of $T$. cruzi strains have been described by different authors (Filardi and Brener, 1987; Toledo et al., 2003; Guedes et al., 2006). Filardi and Brener (1987) described the existence of strains naturally resistant to $\mathrm{Bz}$ and Nf. Recently, Toledo et al. (2003) demonstrated a correlation between phylogenetic divergence among $T$. cruzi stocks and their susceptibilities to chemotherapeutic agents in vivo. Their data indicated that failure in clinical treatment, at least during the acute phase of infection, is most likely related to the T. cruzi strain and its resistance to the specific drugs. However, during the chronic phase, it is more difficult to correlate the parasite strain with drug resistance due to 
the low cure rates obtained. Only $27.1 \%$ of the $T$. cruzi strain isolated from different hosts and geographic areas have been considered resistant to $\mathrm{Bz}$ and/or $\mathrm{Nf}$ (Andrade et al., 1985; Andrade et al., 1992). Clinical trials have shown that treatment with $\mathrm{Bz}$ or $\mathrm{Nf}$ shows small or no effect in prevalent long-term Chagas disease, with cure rates varying among 0-19\% (de Ferreira, 1990; Viotti et al., 1994; Suasnábar et al., 2000; Lauria-Pires et al., 2000; Braga et al., 2000; Cançado, 2002).

More recently, Veloso et al. (2001) showed that longterm infection in vertebrate host can alter $T$. cruzi susceptibility to Bz. In this work, two T. cruzi isolates obtained from dogs inoculated with Be-78 strain, which is $100 \%$ susceptible (Toledo et al., 1995) to Bz, showed $30-50 \%$ resistance to the drug after 25 blood passages in mice. The results indicated that long-term maintenance of $T$. cruzi strains in vertebrate host may induce parasite drug resistance and can explain, at least in part, the therapeutic failure observed for patients treated with $\mathrm{Bz}$ or $\mathrm{Nf}$ during the chronic phase of Chagas disease.

To confirm this hypothesis we studied five T. cruzi populations isolated from dogs inoculated with Be-62 and Be78 strains (sensitive to $\mathrm{Bz}$ ) 2-10 years ago. Immediately after isolation, $\mathrm{Bz}$ sensitivity was determined using the mouse as experimental model. Soon afterwards these stocks were maintained by successive blood passages in mice during 2.5 years, and to each 5 or 10 blood passages a new determination to $\mathrm{Bz}$ sensitivity was performed. The main goal was to evaluate if the type of maintenance would induce new alterations in Bz sensitivity pattern for each $T$. cruzi stock isolated from dogs.

\section{Materials and methods}

\subsection{T. cruzi stocks and their maintenance}

Be-62 and Be-78 strains-T. cruzi II. These strains were isolated from patient Berenice in 1962 (Salgado et al., 1962) and 1978 (Lana and Chiari, 1986), respectively, and maintained cryopreserved in liquid nitrogen as well as by successive blood passages in mice. Berenice is considered to be the first reported human case of Chagas disease (Chagas, 1909).

Five $T$. cruzi stocks were obtained from different outbreed dogs after long-term infection with either Be-62 or Be-78 strains. Two dogs had been inoculated with Be-62 strain 10 years ago (Be-62A and Be-62B), and three had been infected with Be-78 strain for two (Be-78D), seven (Be-78C) and ten (Be-78E) years. Table 1 shows details about the origin of the $T$. cruzi stocks.

The parasites isolated from dogs were grown in LIT (Liver Infusion Tryptose) medium at $28^{\circ} \mathrm{C}$ (Camargo, $1964)$ and inoculated in Swiss mice. Blood from two mice infected with each stock and with wild-type strains (Be-62 and $\mathrm{Be}-78$ ) was collected from orbital venous sinus and inoculated by intraperitoneal route in normal mice. These stocks were maintained in mice for 60 blood passages dur- ing a period of approximately 2.5 years. Blood from the 1st, 5th, 10th, 15th, 20th, 30th, 40th, 50th and 60th blood passage in mice was used to inoculate groups of 16 female albino Swiss mice (about $18-20 \mathrm{~g}$ each) with $5 \times 10^{3}$ bloodstream forms of each stock. Bz resistance profile and parasitemia curves were then evaluated. In parallel was evaluated the $\mathrm{Bz}$ resistance profile and parasitemia curves of Be-62 and Be-78 wild-type strains.

All procedures and experimental protocols were conducted in accordance with the COBEA (Brazilian School of Animal Experimentation) and behavior instructions for the use of animals in research.

\subsection{Determination of the Bz susceptibility}

Bz ( $N$-benzyl-2-nitro-1-imidazolacetamide-Roche Company) resistance/susceptibility tests were performed in groups of 10 mice infected with parasites obtained from the different blood passages. Animals were treated after detection of parasitemia, which occurred around the 4th to the 10th day post-inoculation. $\mathrm{Bz}$ was administered orally $(100 \mathrm{mg} / \mathrm{kg}$ of body weight) in a suspension made with $4 \%$ arabic gum, for 20 consecutive days. Theses stocks were classified according to Toledo et al. (2003) as resistant (cure rates $\leqslant 33 \%$ ), partially resistant $(34 \leqslant$ cure rates $\leqslant$ $66 \%$ ), and susceptible (cure rates $\geqslant 67 \%$ ) to $\mathrm{Bz}$.

\subsection{Evaluation of parasitological cure}

To verify the occurrence of parasitological cure, a battery of four independent tests was performed, including fresh blood examination before and after cyclophosphamide immunosuppression, hemoculture, PCR and a serological enzyme-linked immunosorbent assay. The therapeutic failure was based on both parasitological and serological methods. Animals presenting positive results in the parasitological and serological tests were considered non-cured.

\subsubsection{Fresh blood examination and cyclophosphamide immunosuppression}

Parasitemia of the animals was evaluated between the 15th and 25th day post-treatment to determine the natural reactivation of infection. Animals that did not present reactivation of parasitemia after treatment were submitted to immunosuppression with cyclophosphamide (Genuxal), which consisted of three cycles of $50 \mathrm{mg}$ of cyclophosphamide $/ \mathrm{kg}$ of body weight, for four consecutive days, with intervals of three days between each cycle. Parasitemia of these animals was evaluated during the immunosuppression cycles, as well as for the following 5 days after the end of the treatment.

\subsubsection{Hemoculture}

Thirty days after the end of treatment mice that were not positive by fresh blood examination, before and after cyclophosphamide immunosuppression, were bled from 
Table 1

Origin of the Trypanosoma cruzi stocks studied

\begin{tabular}{lllcll}
\hline Dog & Wild-type strain & Source of the trypomastigote & Time of infection (years) & Clinical form of disease in dog & $T$. cruzi stocks \\
\hline 1 & Be-62 & Metacyclic $^{\text {a }}$ & 10 & Indeterminate \\
2 & Be-62 & Metacyclic $^{\text {a }}$ & 10 & Indeterminate \\
3 & Be-78 & Blood & 7 & Indeterminate \\
4 & Be-78 & Blood & 2 & Indeterminate \\
5 & Be-78 & Metacyclic & Cardiac & Be-62B \\
\hline
\end{tabular}

${ }^{\text {a }}$ Infected triatomine feces and urine

the orbital venous sinus and $400 \mu \mathrm{L}$ of blood was collected and divided into two tubes containing $3 \mathrm{~mL}$ of LIT medium. The tubes were incubated at $28^{\circ} \mathrm{C}$ for 90 days and examined monthly for parasite detection.

\subsubsection{PCR assay}

Mice were bled from the orbital venous sinus and $200 \mu \mathrm{L}$ of blood were collected 30 days after the end of treatment. PCR was performed only in samples from animals with negative fresh blood examination, before and after cyclophosphamide immunosuppression, and hemoculture. DNA extraction and PCR was performed according to Gomes et al. (1998) with some modifications. The primers used for the parasite minicircle amplification were: S35 5' - AAATAATGTACGGG(T/G)GAGATGCATGA-3' and S36 5'-GGGTTCGATTGGGGTTGGTGT-3' (Ávila et al., 1990). Thirty-five cycles of amplifications were carried out in a Research Programmable Thermal Controller (MiniCycler). The cycles consisted of an initial denaturation of $5 \mathrm{~min}$ at $95^{\circ} \mathrm{C}$ followed by 35 cycles of $1 \mathrm{~min}$ at $95^{\circ} \mathrm{C}$ for denaturation, $1 \mathrm{~min}$ at $65^{\circ} \mathrm{C}$ for primer annealing and $1 \mathrm{~min}$ at $72{ }^{\circ} \mathrm{C}$ for primer extension. All the stages were carried out in separate environments with reagents, material, and equipment exclusive to each area. Five microliters of the PCR product was analyzed by electrophoresis on a $6 \%$ polyacrylamide gel and visualized by silver staining (Santos et al., 1993).

\subsubsection{Serological assay}

Blood from treated mice was collected from orbital venous sinus $(0.5 \mathrm{~mL})$ in the $1 \mathrm{st}$ and 6 th month after treatment. T. cruzi specific antibodies were detected by the technique described by Voller et al. (1976). Enzyme-linked immunosorbent assay plates were coated with $T$. cruzi antigen prepared from alkaline extraction of the $T$. cruzi $\mathrm{Y}$ strain at exponential growth in LIT medium. Anti-mouse IgG-peroxidase conjugated antibody (Sigma Chemical Co.) was used. The mean absorbance for 10 negative control samples plus two standard deviations were used as the cut-off to discriminate positive and negative results.

\subsection{Parasitemia curves}

Groups of six mice were inoculated with each $T$. cruzi stock and the parasitemia was evaluated by examination of fresh blood collected from the mouse tail, starting from day 4th post infection. The number of parasites was calculated as described by Brener (1962). Curves were plotted using the mean of the parasitemia obtained from six mice. Mortality rate was cumulative and expressed as a percentage of deaths within the period of 120 days after inoculation.

\subsection{Statistical analysis}

The area under the parasitemia curve was compared using the non-parametric Kolmogorov-Smirnov test. Pearson's correlation $(r)$ was used to evaluate the association between the degree of resistance to $\mathrm{Bz}$ of each $T$. cruzi stock and the number of blood passages in mice. Values of $P<0.05$ were considered significant.

\section{Results}

\subsection{Bz susceptibility of Be-62 and Be-78 stocks after long- term infection in vertebrate host}

The in vivo trypanocidal effect of $\mathrm{Bz}$ for $\mathrm{Be}-62$ and $\mathrm{Be}-78$ stocks in comparison with wild-type strains are show in Fig. 1. Mice infected with wild-type strains, Be-62 or Be78 , showed $100 \%$ cure after treatment with Bz. In contrast, immediately after the isolation from long-term infected dogs, all T. cruzi stocks showed some level of Bz resistance. Mice inoculated with Be-62A and Be-62B, showed 50\% and

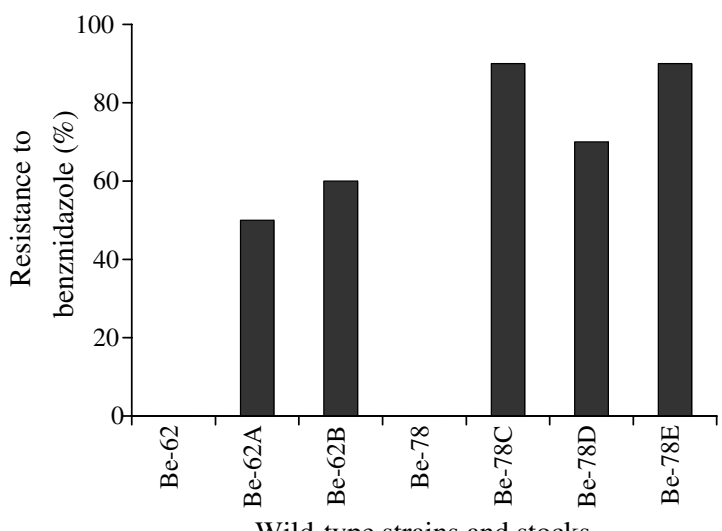

Fig. 1. Percentages of benznidazole resistance in Be-62 and Be-78 wildtype Trypanosoma cruzi strains and Be-62A and B, and Be-78C, D and E stocks, isolated from dogs with long-term infection, in the first blood passage in mice. 
$60 \%$ resistance to $\mathrm{Bz}$, while mice inoculated with $\mathrm{Be}-78 \mathrm{C}$, Be-78D and Be-78E stocks were, respectively, 90\%, 70\% and $90 \%$ resistant to the drug.

\subsection{Bz susceptibility of T. cruzi isolates during successive blood passages in mice}

The effectiveness of each method used for control of cure and detection of therapeutic failure is shown in Fig. 2. Among treated and non-cured animals about 54\% were positive for the fresh blood examination test, before $(10.36 \%)$ or after $(43.52 \%)$ cyclophosphamide immunosuppression. For those negative samples in the fresh blood examination test, $28 \%$ were positive for the hemoculture and $18 \%$ of the remaining were positive for the PCR. The results of serological assays were concordant with the parasitological tests, where all animals that showed at least one positive parasitological test were also positive for the serological test.

The results for Bz sensitivity of T. cruzi stocks after successive blood passages in mice, using the four cure control methods previously described, are shown in Fig. 3.

Be-62A and Be-62B stocks were classified as partially resistant after isolation from dogs. For Be-62A an increase in $\mathrm{Bz}$ resistance level was observed after the 20th and 30th blood passages in mice, but these levels returned to initial values after the 50th passage and remained stable until the 60th blood passage. Be-62B, on the other hand, became resistant to $\mathrm{Bz}$ after the 50th blood passage in mice. Be-78C stock, showed the same Bz resistance pattern after isolation from dogs and during the whole period of evaluation. On the other hand, Be-78D and Be-78E stocks showed an alternation between $\mathrm{Bz}$ resistant and sensitive phenotypes during the successive blood passages in mice. These two stocks were classified as $\mathrm{Bz}$ resistant right after long-term infection in dogs. However, after $20-40$ passages in mice they became sensitive to $\mathrm{Bz}$ like the wild-type strains.

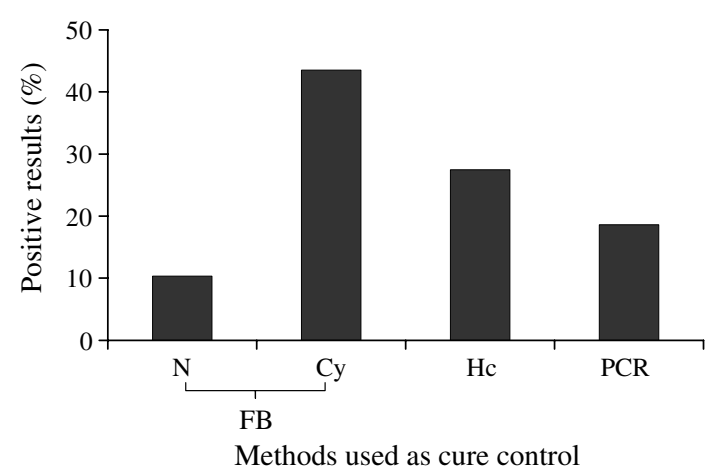

Fig. 2. Positive results obtained with four different methods used as cure control: Fresh blood examination (FB) [Natural parasitemia recrudescence $(\mathrm{N}): 10.36 \%(20 / 193)$ and Parasitemia recrudescence after cyclophosphamide immunosuppression (Cy): 43.52\% (84/193)]; Hemoculture (Hc): $27.46 \%$ (53/193); Polymerase Chain Reaction (PCR): 18.6\% (36/ 193).
The lines in Fig. 3 show two tendencies in Bz resistance pattern during maintenance by successive blood passages (BP) in mice: (1) stabilization of $\mathrm{Bz}$ resistance level in Be$62 \mathrm{~A}\left(50-40 \%\right.$ in $\left.60 \mathrm{BP} ; R^{2}=0.0037\right)$, Be-62B $(60-80 \%$ in $\left.60 \mathrm{BP}, R^{2}=0.1633\right)$ and $\mathrm{Be}-78 \mathrm{C}(90-90 \%$ in $60 \mathrm{BP}$; $R^{2}=0.3$ ); and (2) reduction of $\mathrm{Bz}$ resistance level in Be$78 \mathrm{D}\left(70-20 \%\right.$ in $\left.60 \mathrm{BP} ; R^{2}=0.5647\right)$ and $\mathrm{Be}-78 \mathrm{E}$ (90 to $10 \%$ in $\left.60 \mathrm{BP} ; R^{2}=0.7487\right) T$. cruzi stocks.

\subsection{Biological characterization}

Our results show significant biological differences between Be-62 wild-type strain and its stocks. Fig. 4 shows the parasitemia curves obtained in mice inoculated with Be-62 strain and its two stocks after 1, 20, 40 and 60 blood passages in mice. All isolates showed a parasitemia curve with one distinct peak. However, the wildtype strain, Be-62, induced higher parasitemia levels in relation to the $T$. cruzi stocks $\mathrm{Be}-62 \mathrm{~A}$ and $\mathrm{Be}-62 \mathrm{~B}$ $(P<0.001)$. A tendency of parasitemia increase was observed for all stocks after 40 blood passages in mice, being it statistically significant only in parasitemia curves obtained from mice infected with Be-62B stock $(P<0.005)$. Concerning the infectivity and patent period, no considerable differences were observed among the wild-type strain and its stocks. On the other hand, outstanding reduction was observed in the mortality rate induced by Be-62A and Be-62B stocks $(0-16 \%)$, after one blood passage, in relation to Be-62 wild-type strain $(100 \%)$. However, a tendency to increase the mortality rate after successive blood passages was also observed, being it $16 \%$ and $33 \%$ after the 1 st and 60 th blood passage for Be-62A, and $0 \%$ and $33 \%$ for Be-62B.

Fig. 5 shows the parasitemia curves in mice inoculated with Be-78 wild-type strain and its three stocks. Two types of parasitemia curves were observed. The first one was obtained for Be-78 wild-type strain as well as Be-78C and Be-78E stocks, where two distinct peaks could be detected. Be-78C, however, showed higher parasitemia levels after 20 blood passages in mice in relation to Be-78 wild-type strain and Be-78E stock $(P<0.001)$. The second one was obtained for Be-78D stock, which showed a rather flat curve. In spite of the presented differences, all stocks showed significant increase in parasitemia levels after the 20th or 40th blood passage, demonstrating a discreet tendency of parasitemia increase for these stocks after successive blood passages in mice.

Concerning the infectivity as well as the pre-patent and patent periods for the wild-type strain and its stocks, no significant differences were observed. Also, the mortality rate was similar among the wild-type strain and its stocks until the 40th blood passage. Curiously though, Be-78D stock showed an increase in the mortality rate during the subsequent blood passages in mice, reaching $50 \%$ of mortality after the 60 th passage, in contrast with $0 \%$ of mortality observed for mice inoculated with Be-78 wild-type strain. 

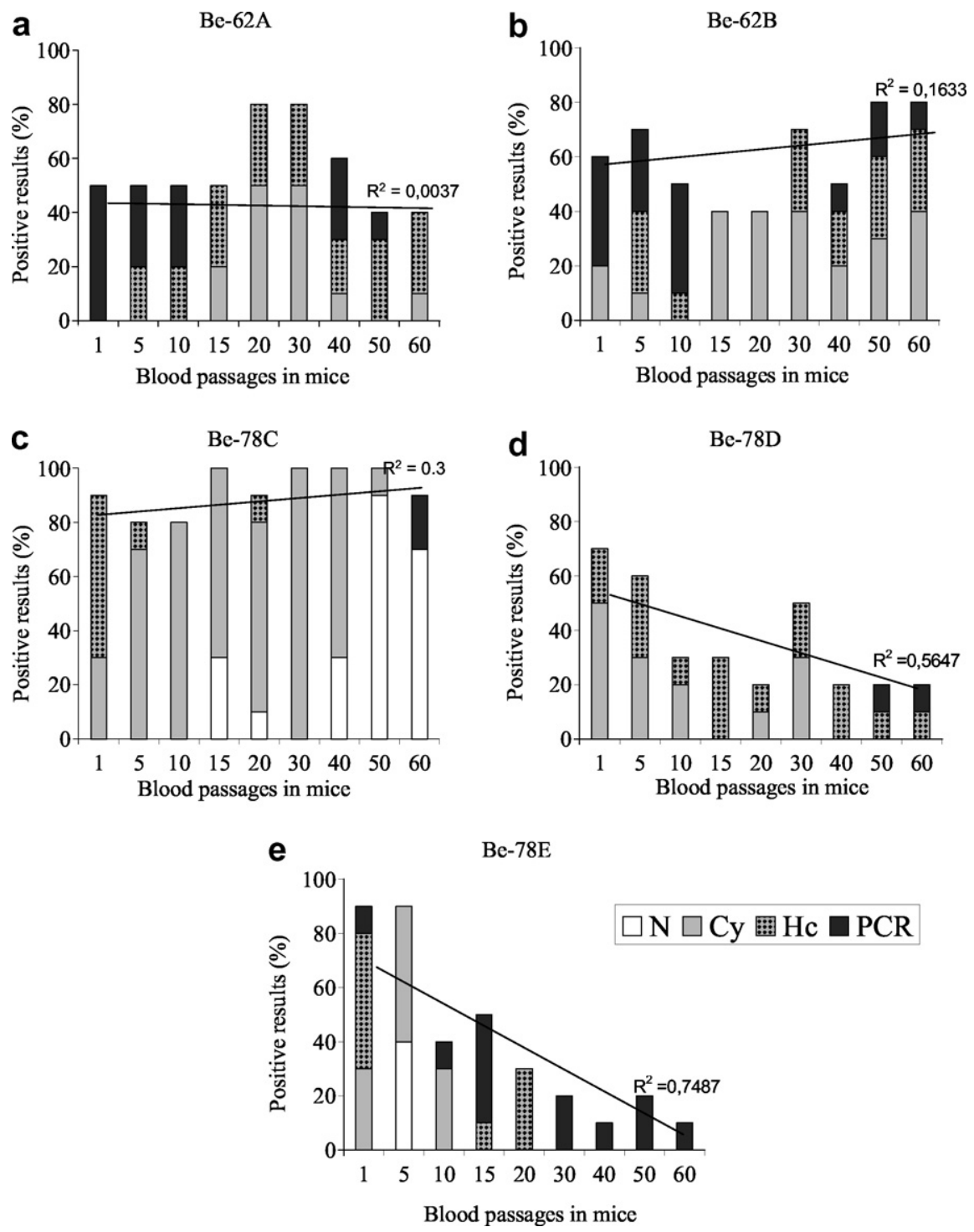

Fig. 3. Results of cure control with four methods of parasites detection-Fresh blood examination (N), Fresh blood examination after cyclophosphamide immunosuppression (Cy), Hemoculture (Hc) and Polymerase Chain Reaction (PCR) in mice infected with Be-62A, Be-62B, Be-78C, Be-78D and Be-78E Trypanosoma cruzi stocks and treated with benznidazole after 1, 5, 10, 15, 20, 30, 40, 50 and 60 blood passages in mice. The lines on the graphs show two tendencies in benznidazole resistance pattern during maintenance by successive blood passages in mice (BP): (a, b and c) stabilization in benznidazole resistance level of Be-62A (50-40\% in $\left.60 \mathrm{BP} ; R^{2}=0.0037\right)$, Be-62B $\left(60-80 \%\right.$ in $\left.60 \mathrm{BP}, R^{2}=0.1633\right)$ and $\mathrm{Be}-78 \mathrm{C}\left(90-90 \%\right.$ in $\left.60 \mathrm{BP} ; R^{2}=0.3\right)$; and $(\mathrm{d}$ and e) reduction of benznidazole resistance level in Be-78D (70-20\% in $\left.60 \mathrm{BP} ; R^{2}=0.5647\right)$ and $\mathrm{Be}-78 \mathrm{E}\left(90-10 \%\right.$ in $\left.60 \mathrm{BP} ; R^{2}=0.7487\right) T$. cruzi stocks.

\section{Discussion}

Drug resistance is a particularly important problem in Chagas disease chemotherapy, but relatively few reports have been published concerning this matter. In vitro and in vivo studies have shown a tendency of $T$. cruzi stocks to increase resistance to clinical drugs. Induction of resistance to fluconazole and $\mathrm{Bz}$ in vitro (Dvorak and Howe, 1977; Buckner et al., 1998; Villarreal et al., 2005), or selection of Bz resistant populations in vivo (Marretto and Andrade, 1994; Murta and Romanha, 1998) by drug pressure have been described. In this work we demonstrated for the first time the induction of $\mathrm{Bz}$ resistance in $T$. cruzi stocks by long-term infection in vertebrate host, even in the absence of drug stress. Therefore, we considered that the drug resistant $T$. cruzi populations, obtained in absence of drug stress, are representative of a mechanism of natural resistance and reflect more closely the situation of field infections.

Five T. cruzi stocks isolated from different dogs previously inoculated (2-10 years ago) with Berenice-62 and Berenice-78, considered $100 \%$ susceptible to $\mathrm{Bz}$ (Toledo et al., 1995), started to show different levels of Bz resistance, and were classified as partially resistant (Be-62A and $\mathrm{Be}-62 \mathrm{~B}$ ) or resistant (Be-78C, D and E) according to the classification proposed by Toledo et al. (2003). The resistance induced by long-term infection in dogs may be related to genetic adaptability of the parasite to the mammalian host. This high level of adaptability allows the parasite to select appropriate strategies to circumvent the 

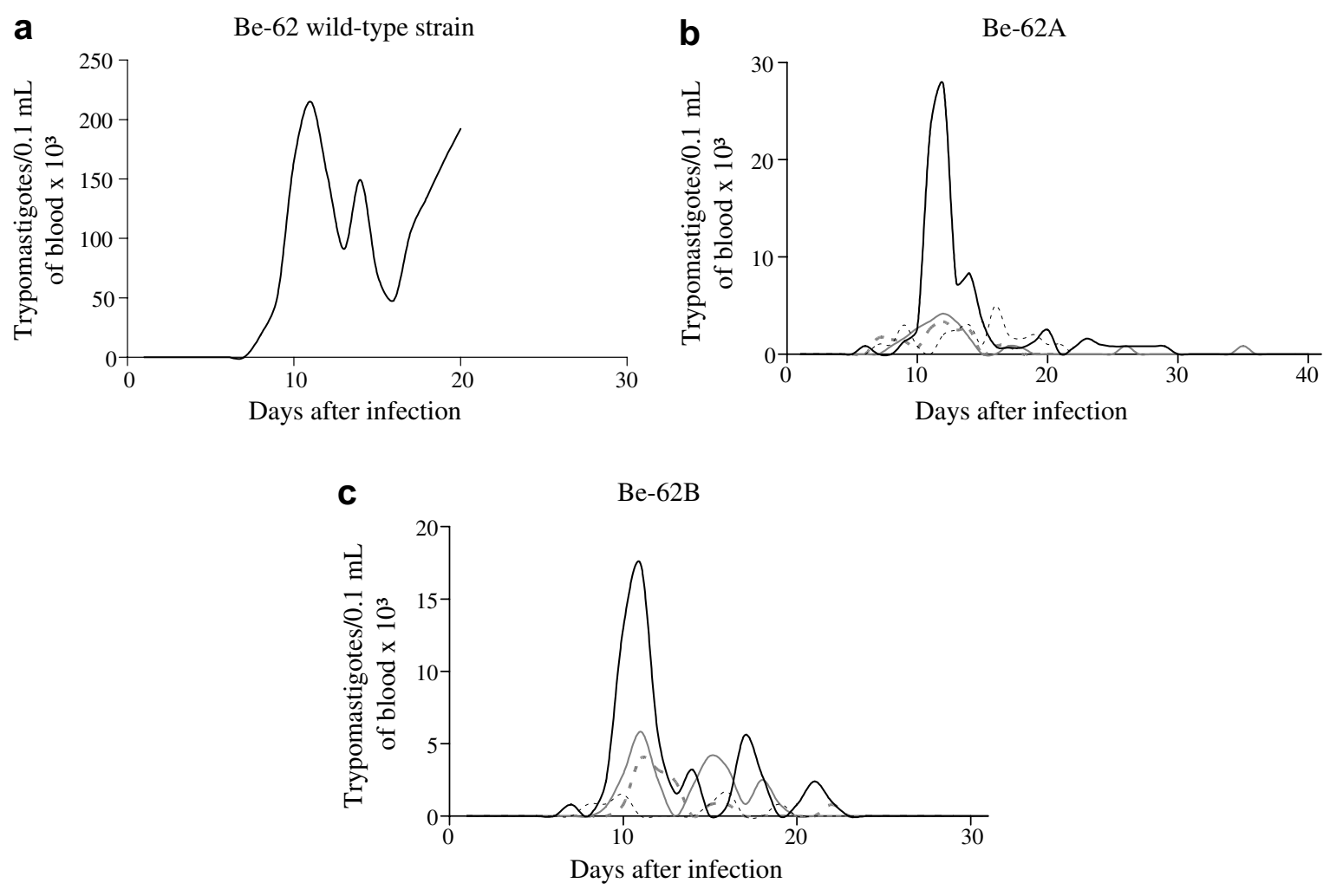

- $=1 \mathrm{BP} \longrightarrow 20 \mathrm{BP} \ldots . . . .40 \mathrm{BP} \longrightarrow 60 \mathrm{BP}$

Fig. 4. Parasitemia curves of Be-62 wild-type strain (a), Be-62A (b) and Be-62B (c) Trypanosoma cruzi stocks, isolated from dogs with long-term infection, after 1, 20, 40 and 60 blood passages in mice (BP). For details see Materials and Methods.
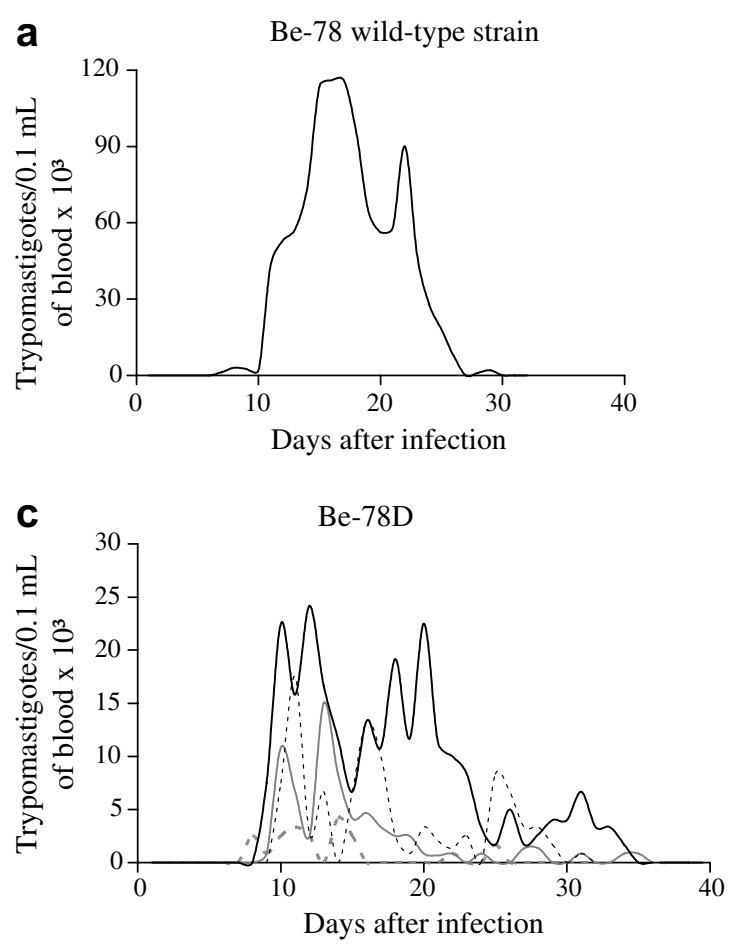
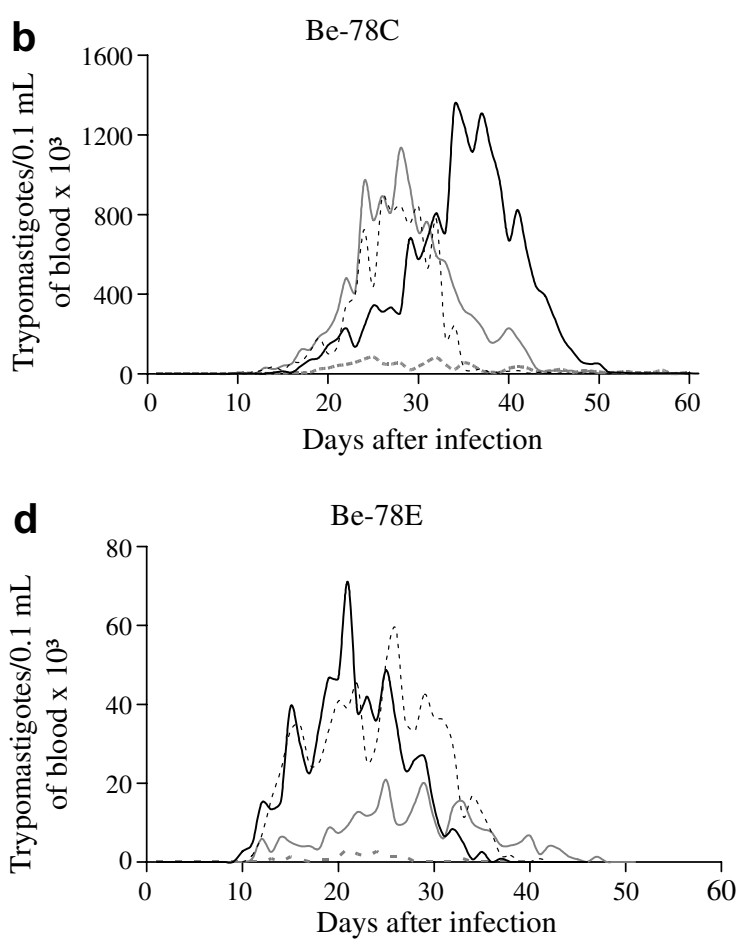

$40 \mathrm{BP}-60 \mathrm{BP}$

Fig. 5. Parasitemia curves of Be-78 wild-type strain (a), and Be-78C (b), Be-78D (c) and Be-78E (d) Trypanosoma cruzi stocks, isolated from dogs with long-term infection, after 1, 20, 40 and 60 blood passages in mice (BP). For details see Materials and methods. 
lethal effects of host immune response, and these alterations may be also important to develop mechanisms of resistance to drugs. Data presented here suggests that $T$. cruzi resistance to Bz may occur in human infections and may explain, at least in part, the low cure indexes observed for individuals with chronic Chagas disease treated with Bz.

The different levels of $\mathrm{Bz}$ resistance found for the studied T. cruzi stocks demonstrate that parasite biological behavior is influenced by interaction with the vertebrate host. These results agree with that obtained by Guedes et al. (2002) that showed the influence of the vertebrate host in the success of $\mathrm{Bz}$ treatment, where $100 \%$ and $53 \%$ of cure was observed in dogs and mice (Filardi and Brener, 1987), respectively, inoculated with the Y strain.

Several authors have shown that $\mathrm{Bz}$ and azole resistance phenotypes are stable in the absence of drug pressure (Buckner et al., 1998; Villarreal et al., 2005). To confirm this hypothesis the resistant and partially resistant $T$. cruzi stocks were maintained by successive blood passages in mice (acute phase) and to each 5 or 10 blood passages the $\mathrm{Bz}$ resistance pattern was determined. Three stocks maintained their resistance pattern. However two stocks became sensitive to the drug after $20-40$ blood passages and remained stable for the following passages, showing that the acquired resistance during long-term infection in vertebrate host can again be altered. It is reasonable to speculate that parasite subpopulations with different genotypes were present in Be-62 and Be-78 stocks, and that $\mathrm{Bz}$ susceptible subpopulations can emerge during successive blood passages in mice becoming the dominant population. These results are supported by previously data (Veloso et al., 2005) where authors detected two distinct genetic groups of parasites in four T. cruzi stocks isolated from different chronic chagasic dogs infected with Be-78 strain. Interestingly, a transition from one genetic group to another was verified when these $T$. cruzi stocks were maintained by blood passages in mice. The authors showed that this transition occurs gradually and that eventually one subpopulation becomes dominant in relation to the other.

Our results reveal that the $T$. cruzi stocks isolated from long-term infected dogs showed significant decrease in virulence for mice in relation to wild-type strains in the initial passages in mice. On the other hand, we detected a considerable increase in parasitemia of all $T$. cruzi stocks during maintenance in mice, occurring usually after the 20th blood passage. Many parasite biological characterization studies have demonstrated that maintenance of $T$. cruzi in mice and long-term chronic infection in human leads to alterations in parasites original biological features (Brener et al., 1974; Lana and Chiari, 1986). Regarding virulence, for instance, both decrease (Brener et al., 1974; Araújo, 2000; Veloso et al., 2005) and intensification (Schlemper, 1982; Carneiro et al., 1991) have been described in experimental infection. Our data support the hypothesis that $T$. cruzi populations present high levels of adaptability to the host, where appropriate strategies are selected to evade the immune response during the acute or chronic phase of the infection. Apparently this phenomenon also is host dependent, because when the different $T$. cruzi stocks were isolated from dogs inoculated with the same $T$. cruzi strains (Be-62 or Be-78) different biological properties were observed.

In this study we also investigated the effectiveness of the induction of parasitemia reactivation by cyclophosphamide immunosuppression in the evaluation of the response to the specific drug treatment. Using this methodology it was possible to detect therapeutic failure in about $54 \%$ of the animals one month post-treatment. From the remaining, $27 \%$ were detected by hemoculture, evaluated up to 60 days and other $18 \%$ by PCR. The sensitivity of the PCR assay to detect $T$. cruzi DNA in blood of treated and non-cured mice not only shows the importance of the PCR assay for assessment of cure, but also strongly supports the use of this technique to assess efficacy of etiological treatment of $T$. cruzi infection. These results are in accordance to Miyamoto et al. (2006) that detected T. cruzi DNA using PCR assay in $100 \%$ of mice infected with $T$. cruzi cloned stocks from genotypes 19, 20, 32 and 39, showing that PCR sensitivity for mice is high and independent of parasite genetic diversity.

The new methodology proposed here for the control of cure was very efficient. The immunosuppression allowed fast and unfailing results in about $43 \%$ of the cases of therapeutic failure and the PCR assay confirmed the doubtful results in the first 45 days after treatment. This allowed a much faster evaluation of treatment efficacy compared to the classical method. The classical method for cure control used by several authors require that animals are evaluated until the sixth month post-treatment for the detection of negative seroconversion and the execution of two hemoculture tests (Filardi and Brener, 1987; Toledo et al., 1997; Veloso et al., 2001; Toledo et al., 2003).

Our results have also shown to be a useful model for the study of the differential gene expression in sensitive and resistant stocks of $T$. cruzi. According to Villarreal et al. (2005), the mechanisms involved in natural drug sensitivity are different from those involved in drug induced chemoresistance, and several different pathways are probably involved in Bz resistance in T. cruzi. Also, Murta et al. (2006) showed that the gene encoding old yellow enzyme is down-regulated in a resistant $T$. cruzi population obtained in vitro, but not in natural $\mathrm{Bz}$ resistant populations or $\mathrm{Bz}$ resistance selected in vivo. It is important to mention that very few information is available about the biochemical mechanisms underlying drug resistance in field isolates of $T$. cruzi and new more informative models are very important to change this scenario.

\section{Acknowledgments}

This work was supported by grants from the Fundação de Amparo à Pesquisa do Estado de Minas Gerais (FAPEMIG), Universidade Federal de Ouro Preto (UFOP), 
Coordenação de Aperfeiçoamento de Pessoal de Nível Superior (CAPES), Conselho Nacional de Desenvolvimento Científico e Tecnológico (CNPq), and a research fellowships from CNPq (Bahia, M.T.).

\section{References}

Andrade, S.G., Andrade, V., Brodskyn, C., Magalhães, J.B., Netto, M.B., 1985. Immunological response of Swiss mice to infection with three different strains of Trypanosoma cruzi. Annals of Tropical Medicine and Parasitology 79, 397-407.

Andrade, S.G., Rassi, A., Magalhães, J.B., Ferriolli, F.F., Luquetti, A.O., 1992. Specific chemotherapy of Chagas disease: a comparison between the response in patients and experimental animals inoculated with the same strains. Transactions of the Royal Society of Tropical Medicine and Hygiene 86, 624-626.

Araújo, I.B., 2000. Estudo Morfobiológico e Isoenzimático de Amostras de Trypanosoma cruzi, Trypanosoma rangeli Isoladas de Casos Humanos, de Vetores e de Mamíferos Silvestres da Região do Médio e Alto Rio Negro-Amazonas, MSc Thesis, Instituto Oswaldo Cruz, Rio de Janeiro.

Aufderheide, A.C., Salo, W., Madden, M., Streitz, J., Buikstra, J., Guhl, F., Arriaza, B., Renier, C., Wittmers Jr., L.E., Fornaciari, G., Allison, M., 2004. A 9,000-year record of Chagas' disease. Proceedings of the National Academy of Sciences of the United States of America 101, 2034-2039.

Ávila, H., Gonçalves, A.M., Nehme, N.S., Morel, C.M., Simpson, L., 1990. Schizodeme analysis of Trypanosoma cruzi stocks from South and Central America by analysis of PCR-amplified minicircle variable region sequences. Molecular and Biochemical Parasitology 42, 175187.

Braga, M.S., Lauria-Pires, L., Argañaraz, E.R., Nascimento, R.J., Teixeira, A.R., 2000. Persistent infections in chronic Chagas' disease patients treated with anti-Trypanosoma cruzi nitroderivatives. Revista do Instituto de Medicina Tropical de São Paulo 42, 157-161.

Brener, Z., 1962. Therapeutic activity and criterion of cure on mice experimentally infected with Trypanosoma cruzi. Revista do Instituto de Medicina Tropical de São Paulo 4, 389-396.

Brener, Z., Chiari, E., Alvarenga, N.J., 1974. Observations on Trypanosoma cruzi strains maintained over an 8-year period in experimentally inoculated mice. Revista do Instituto de Medicina Tropical de São Paulo 16, 39-46.

Buckner, F.S., Wilson, A.J., White, T.C., Van Voorhis, W.C., 1998. Induction of resistance to azole drugs in Trypanosoma cruzi. Antimicrobial Agents and Chemotherapy 42, 3245-3250.

Camargo, E.P., 1964. Growth and differentiation in Trypanosoma cruzi. I. Origin of metacyclic Trypanosomes in liquid media. Revista do Instituto de Medicina Tropical de São Paulo 12, 93-100.

Cançado, J.R., 2002. Long term evaluation of etiological treatment of chagas disease with benznidazole. Revista do Instituto de Medicina Tropical de São Paulo 44, 29-37.

Carneiro, M., Romanha, A.J., Chiari, E., 1991. Biological characterization of Trypanosoma cruzi strains from different zymodemes and schizodemes. Memórias do Instituto Oswaldo Cruz 86, 387-393.

Chagas, C., 1909. Nova tripanosomíase humana: estudos sobre a morfologia e o ciclo evolutivo do Schizotrypanum cruzi n. gen., n. sp., agente etiológico de nova entidade mórbida do homem. Memórias do Instituto Oswaldo Cruz 1, 159-218.

de Ferreira, H.O., 1990. Tratamento da forma indeterminada da doença de Chagas com Nifurtimox e Benzonidazol. Revista da Sociedade Brasileira de Medicina Tropical 23, 209-211.

Dvorak, J.A., Howe, C.L., 1977. The effects of Lampit (Bayer 2502) on the interaction of Trypanosoma cruzi with vertebrate cells in vitro. The American Journal of Tropical Medicine and Hygiene 26, 58-63.

Filardi, L.S., Brener, Z., 1987. Susceptibility and natural resistance of Trypanosoma cruzi strains to drugs used clinically in Chagas disease.
Transactions of the Royal Society of Tropical Medicine and Hygiene 81, 755-759.

Gomes, M.L., Macedo, A.M., Vago, A.R., Pena, S.D., Galvão, L.M., Chiari, E., 1998. Trypanosoma cruzi: optimization of polymerase chain reaction for detection in human blood. Experimental Parasitology 88, 28-33.

Guedes, P.M., Fietto, J.L.R., Lana, M., Bahia, M.T., 2006. Advances in Chagas disease chemotherapy. Anti-Infective Agents in Medicinal Chemistry 5, 175-186.

Guedes, P.M., Veloso, V.M., Tafuri, W.L., Galvão, L.M., Carneiro, C.M., Lana, M., Chiari, E., Soares, K.A., Bahia, M.T., 2002. The dog as model for chemotherapy of the Chagas' disease. Acta Tropica 84, 9 17.

Lana, M., Chiari, C.A., 1986. Comparative biological characterization of Berenice and Berenice-78 strains of Trypanosoma cruzi isolated from the same patient at different times. Memórias do Instituto Oswaldo Cruz 81, 247-253.

Lauria-Pires, L., Braga, M.S., Vexenat, A.C., Nitz, N., Simões-Barbosa, A., Tinoco, D.L., Teixeira, A.R., 2000. Progressive chronic Chagas heart disease ten years after treatment with anti-Trypanosoma cruzi nitroderivatives. The American Journal of Tropical Medicine and Hygiene 63, 111-118.

Marretto, J.P., Andrade, S.G., 1994. Biochemical behavior of Trypanosoma cruzi strains isolated from mice submitted to specific chemotherapy. Revista da Sociedade Brasileira de Medicina Tropical 27, 209 215.

Miyamoto, C.T., Gomes, M.L., Marangon, A.V., Araújo, S.M., Bahia, M.T., Lana, M., Toledo, M.J., 2006. Trypanosoma cruzi: sensitivity of the polymerase chain reaction for detecting the parasite in the blood of mice infected with different clonal genotypes. Experimental Parasitology 112, 198-201.

Murta, S.M., Krieger, M.A., Montenegro, L.R., Campos, F.F., Probst, C.M., Ávila, A.R., Muto, N.H., de Oliveira, R.C., Nunes, L.R., Nirdé, P., Bruna-Romero, O., Goldenberg, S., Romanha, A.J., 2006. Deletion of copies of the gene encoding old yellow enzyme (TcOYE), a $\mathrm{NAD}(\mathrm{P}) \mathrm{H}$ flavin oxidoreductase, associates with in vitro-induced benznidazole resistance in Trypanosoma cruzi. Molecular and Biochemical Parasitology 146, 151-162.

Murta, S.M., Romanha, A.J., 1998. In vivo selection of a population of Trypanosoma cruzi and clones resistant to benznidazole. Parasitology 116 (Pt 2), 165-171.

Salgado, J.A., Garcez, P.N., Olliveira, C.A., Galizzi, J., 1962. Revisão clínica atual do primeiro caso humano descrito de Doença de Chagas. Revista do Instituto de Medicina Tropical de São Paulo 4 (5), 330-337.

Santos, F.R., Pena, S.D., Epplen, J.T., 1993. Genetic and population study of a Y-linked tetranucleotide repeat DNA polymorphism with a simple non-isotopic technique. Human Genetics 90, 655-656.

Schlemper Jr., B.R., 1982. Caracterização de Cepas do Trypanosoma cruzi Isoladas de Pacientes com Diferentes Formas Clínicas da Doença de Chagas, PhD Thesis, Universidade Federal do Rio de Janeiro.

Suasnábar, D.F., Arias, E., Streiger, M., Piacenza, M., Ingaramo, M., Del Barco, M., Amicone, N., 2000. Evolutive behavior towards cardiomyopathy of treated (nifurtimox or benznidazole) and untreated chronic chagasic patients. Revista do Instituto de Medicina Tropical de São Paulo 42, 99-110.

Toledo, M.J., Bahia, M.T., Carneiro, C.M., Martins-Filho, O.A., Tibayrenc, M., Barnabé, C., Tafuri, W.L., Lana, M., 2003. Chemotherapy with benznidazole and itraconazole for mice infected with different Trypanosoma cruzi clonal genotypes. Antimicrobial Agents and Chemotherapy 47, 223-230.

Toledo, M.J., Gasperi, M.V., Marques de Araújo, S., Lana, M., 1995. Berenice 62 and Berenice 78 strains of Trypanosoma cruzi: comparison of their susceptibility to benzidazole. Memórias do Instituto Oswaldo Cruz 90 (suppl.), 201.

Toledo, M.J., Guilherme, A.L., Silva, J.C., de Gasperi, M.V., Mendes, A.P., Gomes, M.L., Marques de Araújo, S., 1997. Trypanosoma cruzi: chemotherapy with benznidazole in mice inoculated with strains from 
Paraná state and from different endemic areas of Brazil. Revista do Instituto de Medicina Tropical de São Paulo 39, 283-290.

Veloso, V.M., Carneiro, C.M., Toledo, M.J., Lana, M., Chiari, E., Tafuri, W.L., Bahia, M.T., 2001. Variation in susceptibility to benznidazole in isolates derived from Trypanosoma cruzi parental strains. Memórias do Instituto Oswaldo Cruz 96, 1005-1011.

Veloso, V.M., Romanha, A.J., Lana, M., Murta, S.M., Carneiro, C.M., Alves, C.F., Borges, E.C., Tafuri, W.L., Machado-Coelho, G.L., Chiari, E., Bahia, M.T., 2005. Influence of the long-term Trypanosoma cruzi infection in vertebrate host on the genetic and biological diversity of the parasite. Parasitology Research 96, 382-389.

Villarreal, D., Nirdé, P., Hide, M., Barnabé, C., Tibayrenc, M., 2005. Differential gene expression in benznidazole-resistant Trypanosoma cruzi parasites. Antimicrobial Agents and Chemotherapy 49, 27012709.

Viotti, R., Vigliano, C., Armenti, H., Segura, E., 1994. Treatment of chronic Chagas' disease with benznidazole: clinical and serologic evolution of patients with long-term follow-up. American Heart Journal 127, 151-162.

Voller, A., Bidwell, D.E., Bartlett, A., 1976. Enzyme immunoassays in diagnostic medicine. Theory and practice. Bulletin of the World Health Organization 53, 55-65.

WHO, 2002. WORLD HEALTH ORGANIZATION. Control of Chagas' disease. Second Report of the WHO Expert Committee. WHO Technical Report Series. 905, Geneva. 